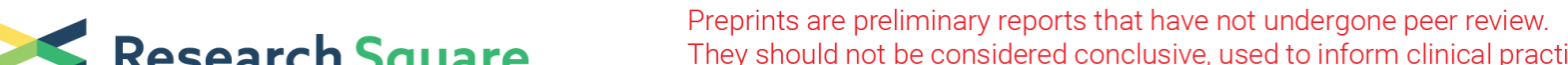 $\begin{array}{ll}\text { Research Square } & \text { They should not be considered conclusive, used to inform clinical practice, } \\ \text { or referenced by the media as validated information. }\end{array}$
}

\section{Network Pharmacological Analysis of Huanglian Jiedu Decoction for Anti- Atherosclerosis}

\section{Kerui Wu}

The Second Affiliated Hospital of Guangzhou University of Chinese Medicine https://orcid.org/00000002-7181-8622

\section{Lu Jiang}

The Second Affiliated Hospital of Guangzhou University of Chinese Medicine

\section{Lanlin Huang}

The Second Affiliated Hospital of Guangzhou University of Chinese Medicine

\section{Yaxing He}

The Second Affiliated Hospital of Guangzhou University of Chinese Medicine

\section{Xia Yan}

The Second Affiliated Hospital of Guangzhou University of Chinese Medicine

Dawei Wang ( $\sim$ davidwang33@139.com )

https://orcid.org/0000-0002-9775-0443

\section{Research}

Keywords: Huanglian Jiedu Decoction, Anti-atherosclerosis, Network Pharmacology

Posted Date: August 5th, 2020

DOI: https://doi.org/10.21203/rs.3.rs-44241/v1

License: (c) (i) This work is licensed under a Creative Commons Attribution 4.0 International License.

Read Full License 


\section{Network Pharmacological Analysis of Huanglian Jiedu Decoction for Anti-Atherosclerosis}

Kerui $\mathrm{Wu}^{1}$,Lu Jiang ${ }^{1}$,Lanlin Huang ${ }^{1}$, Yaxing $\mathrm{He}^{1}$, Xia Yan ${ }^{1}$,Dawei Wang ${ }^{2}$

1. The Second Affiliated Hospital of Guangzhou University of Chinese Medicine,Guangzhou 510006, China

2. Shunde Affiliated Hospital of Guangzhou University of Chinese Medicine,Shunde 528300, China

For Correspondence:Dawei Wang:davidwang33@139.com

Objective:We aimed to predict the possible active components,key targets and pathways of Huanglian Jiedu Decoction(HLJDD) for anti-atherosclerosis. Methods:The TCMSP database was searched to obtain the active components and targets of HLJDD, the GeneCards and OMIM databases were searched to obtain related targets of atherosclerosis, and we obtain the intersection targets of them, which are the potential targets of HLJDD for anti-atherosclerosis.Application of Cytoscape 3.6.0 software to build a herbal-active ingredient-potential target regulation network.We perform protein-protein interaction(PPI) network analysis of potential targets through STRING 11.0 database and obtain the key targets,and the results of PPI network of key targets were visualized by Cytoscape3.6.0 software. Gene ontology (GO) analysis and Kyoto Encyclopedia of Genes and Genomes (KEGG) pathway enrichment analysis of the key targets were performed using STRING11.0 database, and we finally constructed the possible pharmacological network of HLJDD for anti-atherosclerosis.Results:We finally obtained 14 key active ingredients of HLJDD, 65 key targets of anti-atherosclerosis, and 14 key active ingredients corresponded to 52 of these targets. These targets are mainly involved in biological processes such as reaction to organic substance, reaction to chemical stimulation,etc. They mainly involved in biological signaling pathways such as pathways in cancer,IL-17 signaling pathway,etc.Conclusion:HLJDD may act on 52 key targets such as PTGS2, HSP90AA1 and RELA through 14 key active ingredients, and influence the signaling pathways including fluid shear stress and atherosclerosis,PI3K-Akt signaling pathway,IL-17 signaling pathway,AGE-RAGE signaling pathway in diabetic complications, TNF signaling pathway,etc.Thus, it may play an anti-atherosclerosis role by inhibiting inflammatory reaction, oxidative stress and improving hemodynamics, etc.

Key words: Huanglian Jiedu Decoction;Anti-atherosclerosis;Network Pharmacology

\section{Introduction}

As seriously endangering human health and reducing the quality of human life,cardiovascular diseases have currently become one of the main diseases leading to death and disability ${ }^{[1]}$. Over the past ten years, the number of deaths due to cardiovascular disease has increased by $12.5 \%$, which currently accounts for about one-third of the deaths globally, and the number of people with cardiovascular disease is increasing,especially in southeast Asia,which has a large and rapidly growing population ${ }^{[1-2]}$. Therefore, finding a method for preventing and treating cardiovascular disease,blocking the formation or delaying the progression of cardiovascular disease has become a hot issue in current research.

Atherosclerosis(AS) is a chronic inflammatory disease that occurs in the endothelium of arteries,which is considered a potential process of cardiovascular disease ${ }^{[3]}$. Therefore, anti-atherosclerosis is essential for the prevention and treatment of cardiovascular diseases. 
However, the pathogenesis of atherosclerosis has not yet been fully elucidated. Previous studies suggest that its occurrence and development mainly involve multiple pathological processes such as inflammatory response, lipid infiltration, endothelial damage, cell autophagy, smooth muscle cell proliferation, and vascular remodeling,ect ${ }^{[4-6]}$. The inflammatory response theory of AS was proposed by the scholar Ross ${ }^{[7]}$ and has been widely accepted. Inflammatory reaction was involved in the whole process of the occurrence and development of AS.Inflammatory cells and mediators are involved in the damage of vascular endothelial cells, lipid accumulation, foam cell formation, plaque formation, plaque rupture and thrombosis, and the whole development process lacks the transition from proinflammatory to anti-inflammatory, thus forming chronic and persistent inflammation of vascular endothelium ${ }^{[8-9]}$. Therefore, a large number of studies have taken anti-inflammatory as a fucus and tried to find an effective treatment method to reduce the vascular endothelial inflammatory response and protect the vascular endothelium, so as to achieve the purpose of anti-atherosclerosis ${ }^{[10-12]}$.

In traditional Chinese medicine(TCM), heat-clearing and detoxifying Chinese herbal medicines have often been proven to have varying degrees of anti-inflammatory effects. HLJDD is a typical prescription for clearing heat and detoxifying in traditional Chinese medicine,which consists of Coptidis Rhizoma(CR),Scutellariae Radix(SR),Phellodendri Chinrnsis Cortex(PCC) and Gardeniae Fructus(GF).Studies have shown that HLJDD can produce anti-atherosclerotic effect through anti-inflammatory.Zhou et al.found through experiments that HLJDD can significantly reduce aortic plaque injury in Apo E-/- mice, and the mechanism may be related to increasing the number of $\mathrm{T}$ lymphocytes and reducing the level of serum inflammatory cytokines ${ }^{[13]}$.Yu et al. showed that HLJDD can reduce the expression of monocyte chemotactic protein (MCP-1) and vascular cell adhesion molecule (VCAM-1), inhibit the inflammatory response in atherosclerotic rats, and thus interfere with the formation of arterial plaques $^{[14]}$.Moreover, studies has also shown that in addition to being related to anti-inflammatory effects, the anti-atherosclerosis effect of HLJDD is also related to the reduction of blood lipid level, the enhancement of endothelial progenitor cell function and the anti-oxidative stress effect,ect ${ }^{[14-16]}$. Based on the current research background, the mechanism of anti-atherosclerosis of HLJDD has not been fully elucidated, which may be related to the multi-component and multi-target characteristics of Chinese herbal medicine.

Based on the theory that drug action is the result of interaction of effective molecules with multiple targets in a network of biological systems, network pharmacology is proposed as a methodology that combines systems biology and multiple pharmacology,which provides a new network research model of "multi-target, multiple effects, and complex diseases" for drug research ${ }^{[17-18]}$. Chinese herbal medicine has the characteristics of complex ingredients and unclear targets,so network pharmacology is suitable for the study of Chinese medicine pharmacology. Studying the interaction and mechanism of Chinese herbal medicine components and targets in the context of molecular regulatory networks is beneficial to better understand the overall, dialectical, and coordinated characteristics of Chinese herbal medicine ${ }^{[19]}$. Therefore, we tried to apply the method of network pharmacology to study the possible mechanism of anti-atherosclerosis of HLJDD. In this study, we first searched out the active ingredients and targets of HLJDD, and at the same time found the related targets of atherosclerosis. We screened out the possible targets of anti-atherosclerosis of HLJDD through intersection analysis. Performed protein-protein interaction network analysis, screened out key targets, constructed protein-protein interaction network of key 
targets,performed GO analysis and KEGG pathway enrichment analysis of key targets,and constructed the pharmacological network of "HLJDD-key target-pathway".We aim to find out possible key active ingredients, key targets and action pathways of HLJDD for anti-atherosclerosis, so as to provide some references for further research on the effect of HLJDD for atherosclerosis.

\section{Materials and Methods}

2.1 Screening of Active Ingredients and Targets of HLJDD The chemical components of four Chinese herbal medicines including CR, SR, PCC, and GF were obtained by searching Traditional Chinese Medicine Systems Pharmacology Database and Analysis Platform(TCMSP, $\mathrm{http} / / / \mathrm{tcmspw} . c o m / t c m s p . p h p)^{[20]}$. Based on $\operatorname{ADME(absorption,~distribution,~metabolism~and~}$ excretion) prediction model, the active ingredients of HLJDD were screened with oral bioavailability $(\mathrm{OB}) \geq 30 \%$ and drug similarity (DL) $\geq 0.18$. At the same time, we searched TCMSP to obtain all the reported targets of the four Chinese herbal medicines, and a database of targets was constructed. Perl software (https://www.perl.org/get.html)was used to screen out the targets corresponding to the active ingredients, and we construct the file of "HLJDD - Active Ingredients - Targets ".

2.2 Obtaining Gene Name of The Targets The protein name of the target was input into the online database STRING11.0(https://string-db.org/cgi/input.pl) ${ }^{[21]}$,and we converted the protein names into corresponding gene names in the context of setting the species as homo sapiens. Some of the targets that could not get the corresponding gene names were deleted, and finally we constructed the file of "HLJDD- Active Ingredient - Target - Gene".

2.3 Obtaining Related Targets of AS Using "atherosclerosis" as a search term, we searched GeneCards database(https://www.genecards.org/) ${ }^{[22]}$ and OMIM database (https://www.omim.org/) ${ }^{[23]}$ to obtain the related target genes of atherosclerosis.

2.4 Screening of The Potential Targets of HLJDD for Anti-atherosclerosis Based on the targets of the active ingredients of HLJDD and the related targets of atherosclerosis, R 3.6.1 software (https://www.r-project.org/) was used to obtain the common targets of the active ingredients of HLJDD and atherosclerosis, which was considered as a potential target of HLJDD for anti-atherosclerosis.

\subsection{Construction of "HLJDD-Active Ingredient-Potential Targets " regulation network} Perl software was applied to identify the Chinese herbal medicine and its active components corresponding to the potential targets of HLJDD for anti-atherosclerosis, and the file of "HLJDD-active ingredient-potential targets" was constructed. Cytoscape3.6.0 software was used to construct the regulation network of "HLJDD-Active Ingredient-Potential Targets". Through topological analysis of the network, the active components with node connection degree greater than the average node connection degree of the network were found out and identified as the key active components of HLJDD for anti-atherosclerosis.

2.6 Construction of Protein-Protein Interaction(PPI) Network of The Potential Targets Enter the gene name of the potential targets into the online database STRING11.0, set the species as homo sapiens, confidence score $\geq 0.4$, do not display free targets, we finally obtain PPI network of the potential targets and corresponding result files. The greater the node connection degree of each target in the network, it indicates that it has a more important position in the network and has a higher probability to become a key target.Analyzing the network, the protein that the node connection degree is greater than the average node connection degree is obtained, which is 
considered as the key node of the network, that is, the key targets. R 3.6.1 software was used to visualize the key targets and their corresponding node connection degree. The gene names of the key targets were imported into the online database STRING11.0 again, and the PPI network analysis of the key targets was performed in the same operation method.The results were exported in text format, and the PPI network was visualized by Cytoscape3.6.0 software.

2.7 GO Analysis And KEGG Pathway Enrichment Analysis of The Key Targets Application of the analysis function in the online database STRING11.0, the GO analysis and KEGG pathway enrichment analysis of key targets were performed, and the false discovery rate(FDR) $\leq 0.05$ was considered to be of statistical significance. The "ggplot2" package of R 3.6.1 software was used to visualize the GO analysis and KEGG pathway enrichment analysis results.

2.8 Construction of "HLJDD-Key Active Ingredient-Key Targets-Pathway" network Based on the results of key active ingredients, key targets and KEGG pathway enrichment analysis, the network of "HLJDD-Key Active Ingredient-Key Targets-Pathway" was constructed by using Cytoscape3.6.0 software.

\section{Result}

3.1 Active Ingredient and Targets Screening of HLJDD By screening the TCMSP database, a total of 429 ingredients were retrieved, which are as follow:CR,48;SR,143;PCC,140;GF,98. According to $\mathrm{OB}$ and DL, 102 active ingredients were obtained, including 14 of CR, 36 of SR, 37 of PCC, and 15 of GF(supplementary Table1). In addition, a total of 248 corresponding targets were obtained.Finally,a total of 222 gene names corresponding to the targets were found in the STRING database(supplementary Table2).

3.2 The Related Target of Atherosclerosis Through screening the GeneCards and OMIM databases, we obtained in total of 3812 related targets of atherosclerosis(supplementary Table 3).

3.3 The Potential Targets Screening HLJDD for Anti-atherosclerosis The R 3.6.1 software was used to obtain the intersection of the targets of active ingredient of HLJDD and the related targets of atherosclerosis, and finally 175 common targets were screened, as shown in Figure 1. The blue part of picture stands for the targets of active ingredient of HLJDD, and the yellow one represents the related targets of atherosclerosis, the purple one stands for the common targets, which means the potential targets of HLJDD for anti-atherosclerosis.

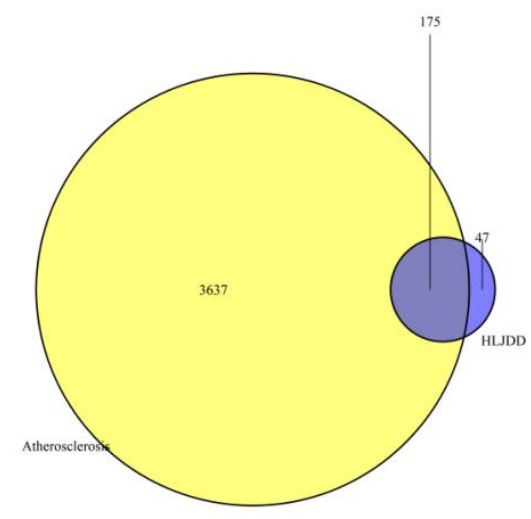

Figure1. Vene figure of Screening Potential Targets of HLJDD for Anti-atherosclerosis 3.4 The regulatory network of "HLJDD-active ingredient-potential target" Based on the established file of "HLJDD-active ingredient-potential target", we constructed a regulatory network, as shown in Figure 2. The regulatory network contains 4 Chinese herbal medicine nodes 
(yellow nodes), 65 active ingredient nodes (green nodes), 175 target nodes (blue nodes), 78 associations of "Chinese herbal medicine-active ingredient" and 1262 associations of "active ingredient-potential target". The 65 active ingredients may play an anti-atherosclerotic role by acting on 175 targets. A topological analysis of the regulatory network shows that the average degree value of the active ingredients was 19.42, and there were 14 active ingredients with degree value $\geq 20$, as shown in Table 1 . These active ingredients play an important role in the network, suggesting that they may be the key active ingredients of HLJDD for anti-atherosclerosis.

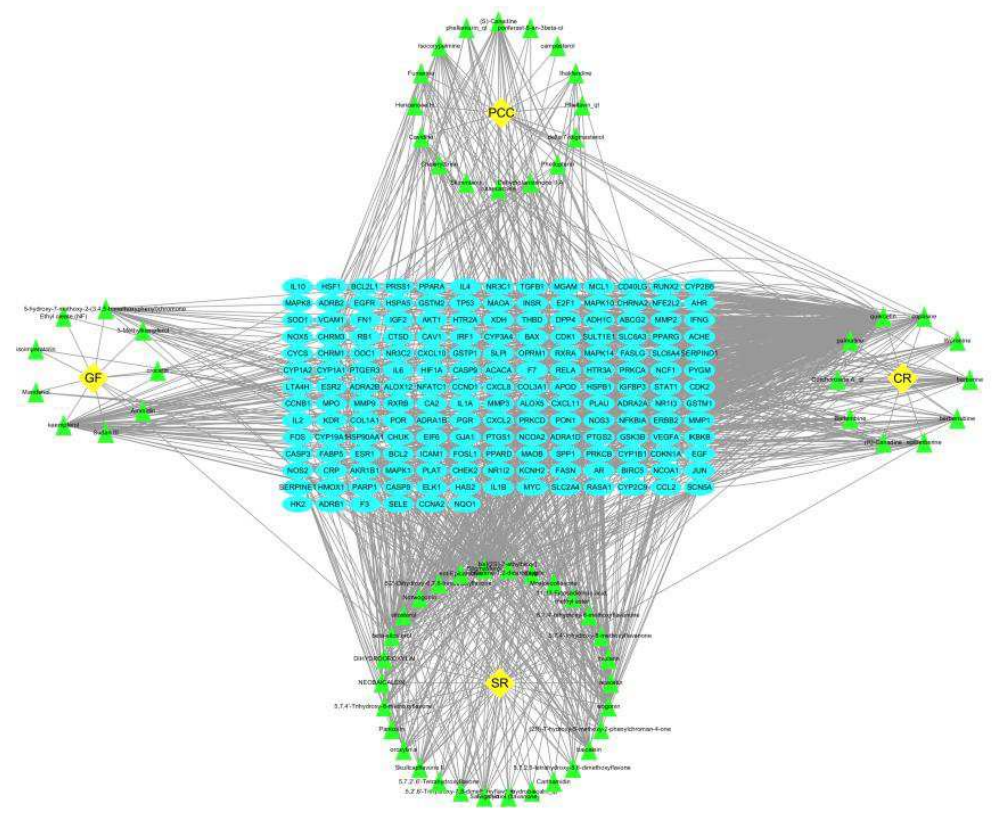

Figure2. HLJDD-Active Ingredient-Target Regulation Network

Table1.Active ingredients with a node connection degree equal to or greater than $\mathbf{2 0}$

\begin{tabular}{cc}
\hline Active ingredients & Node connection degree \\
\hline quercetin & 381 \\
beta-sitosterol & 75 \\
Stigmasterol & 69 \\
kaempferol & 48 \\
wogonin & 36 \\
palmatine & 30 \\
baicalein & 30 \\
berberine & 26 \\
coptisine & 24 \\
Isocorypalmine & 22 \\
acacetin & 21 \\
(S)-Canadine & 21 \\
berberrubine & 20 \\
oroxylin a & 20 \\
\hline
\end{tabular}

3.5 PPI network of the key targets of HLJDD for anti-atherosclerosis The gene names of 175 potential targets were entered into the online database STRING11.0 for PPI network analysis, 
and build a PPI network,as shown in supplementary Figure1. The analysis of the network shows that the network consists of 175 target nodes and 3369 associations and the average node degree of network is 38.05. The results are exported in text format. R 3.6.0 software is used for statistics, and targets with node degree $>38.5$ in the network are screened out. Finally, 65 targets were obtained, and the node connection degrees of each target node were shown in Figure 3. These targets play a key role in the PPI network, indicating that they may be the key targets of HLJDD for anti-atherosclerosis. Then we performed the same operation for the PPI network analysis of 65 key targets, using the Cytoscape software to visualize the results of PPI network,as shown in Figure 4 . The larger the node in the figure, the greater the node connection degree, and the more targets associated with it.

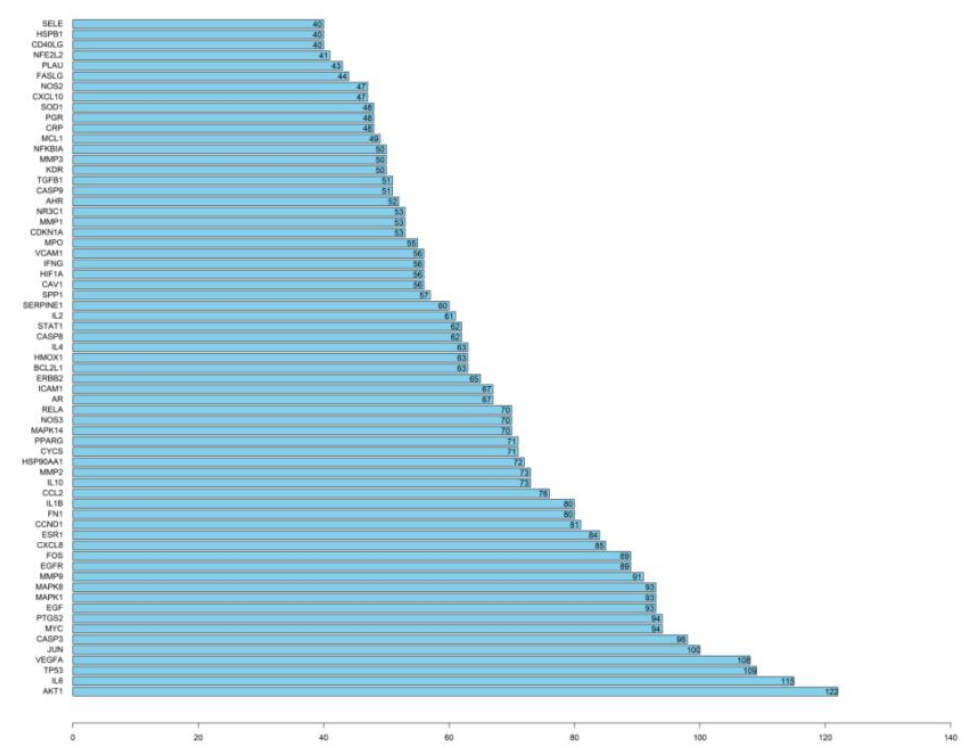

Figure3.65 Key Targets and Their Node Connection Degree

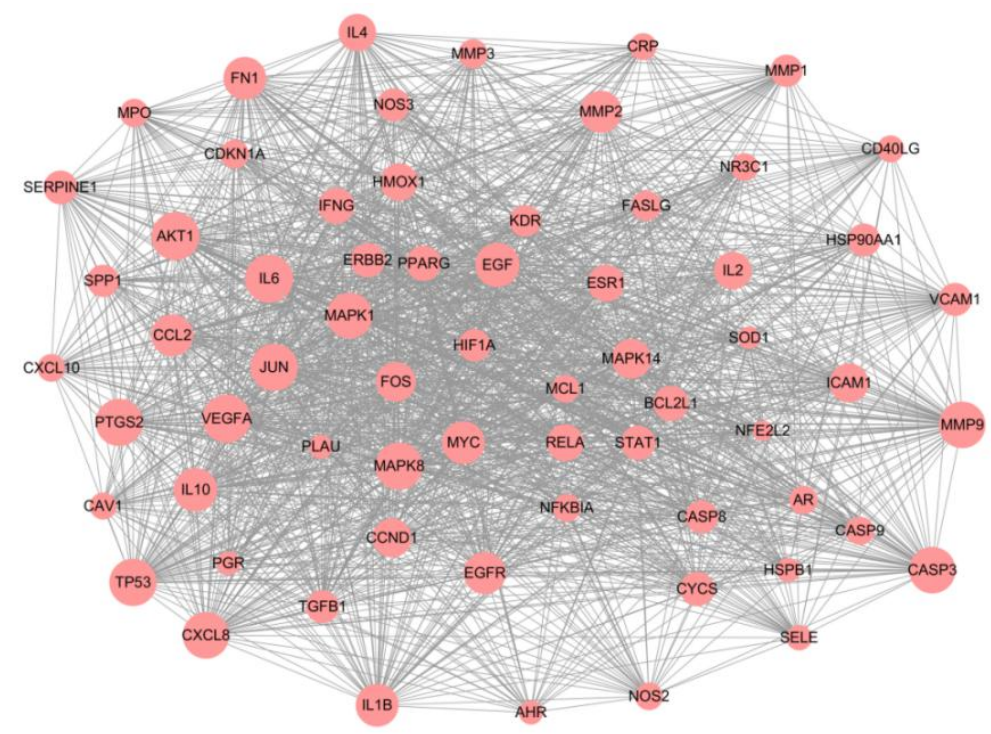

Figure4.PPI Network of Key Targets of HLJDD for Anti-atherosclerosis

3.6 GO Analysis And KEgG Pathway Enrichment Analysis The gene names of 65 key targets were entered into the database STRING11.0, and the analysis function provided by it was used for GO analysis, mainly involving biological process (BP).The results indicated that 65 key 
targets mainly involved $1451 \mathrm{BP}$, such as response to organic substance, response to chemical stimulus, response to cytokines, response to oxygen-containing compound, positive regulation of cellular process, regulation of cell population proliferation, and response to lipids.KEGG pathway enrichment analysis indicated that HLJDD may act on 157 signaling pathways including pathways in cancer,IL-17 signaling pathway,AGE-RAGE signaling pathway in diabetic complications, fluid shear stress and atherosclerosis, and TNF signaling pathway,ect. R 3.6.0 software was applied to visualize the first $20 \mathrm{GO}$ terms and KEGG pathways, and the results were shown in figure 5 and figure 6. The results of enrichment analysis indicate that HLJDD may play a synergistic role in anti-atherosclerosis by regulating multiple biological processes and signaling pathways.

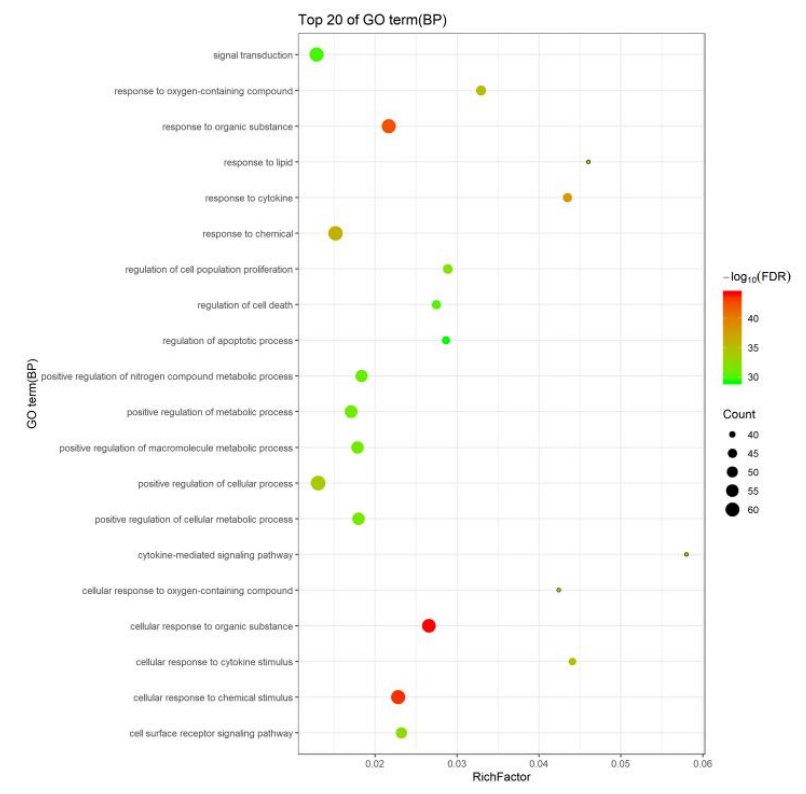

Figure5.GO Analysis(Biological Process) of Key Targets of HLJDD for Anti-atherosclerosis

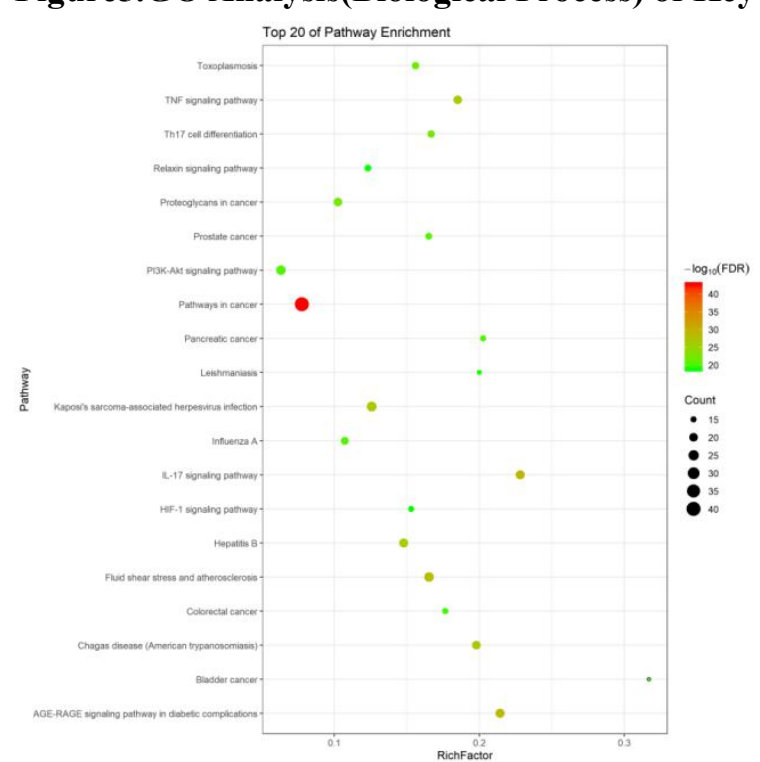

Figure6.KEGG Pathway Enrichment Analysis of Key Targets of HLJDD for Anti-atherosclerosis

3.7 HLJDD-Key Active Ingredients-Key Targets-Pathway Network 4 Chinese herbal medicines, 14 key active ingredients, 65 key targets, and the pathways of the top 20 KEGG enrichment pathways with enrichment targets $>20$ were included in the network construction, and 
the targets unassociated with the key active ingredients and pathways were deleted, as shown in Figure 7. The network consists of 4 Chinese herbal medicine nodes(yellow prismatic nodes), 14 key active ingredient nodes (green triangle nodes), 52 key target nodes (pink oval nodes), and 10 pathway nodes (blue hexagon nodes). The analysis of the network shows that among the 14 key active ingredients, quercetin is the node with the highest connection degree, with 47 targets connected, and Isocorypalmine and (S) -Canadine are the nodes with the lowest connection degree, with 2 targets respectively. Among the 52 targets, the top 3 with the highest node connection degree are PTGS2, HSP90AA1, and RELA. PTGS2 is connected to 14 ingredient nodes and 4 pathway nodes, and HSP90AA1 is connected to 11 ingredient nodes and 4 pathway nodes, while RELA is connected to 5 ingredient nodes and 9 pathway nodes.

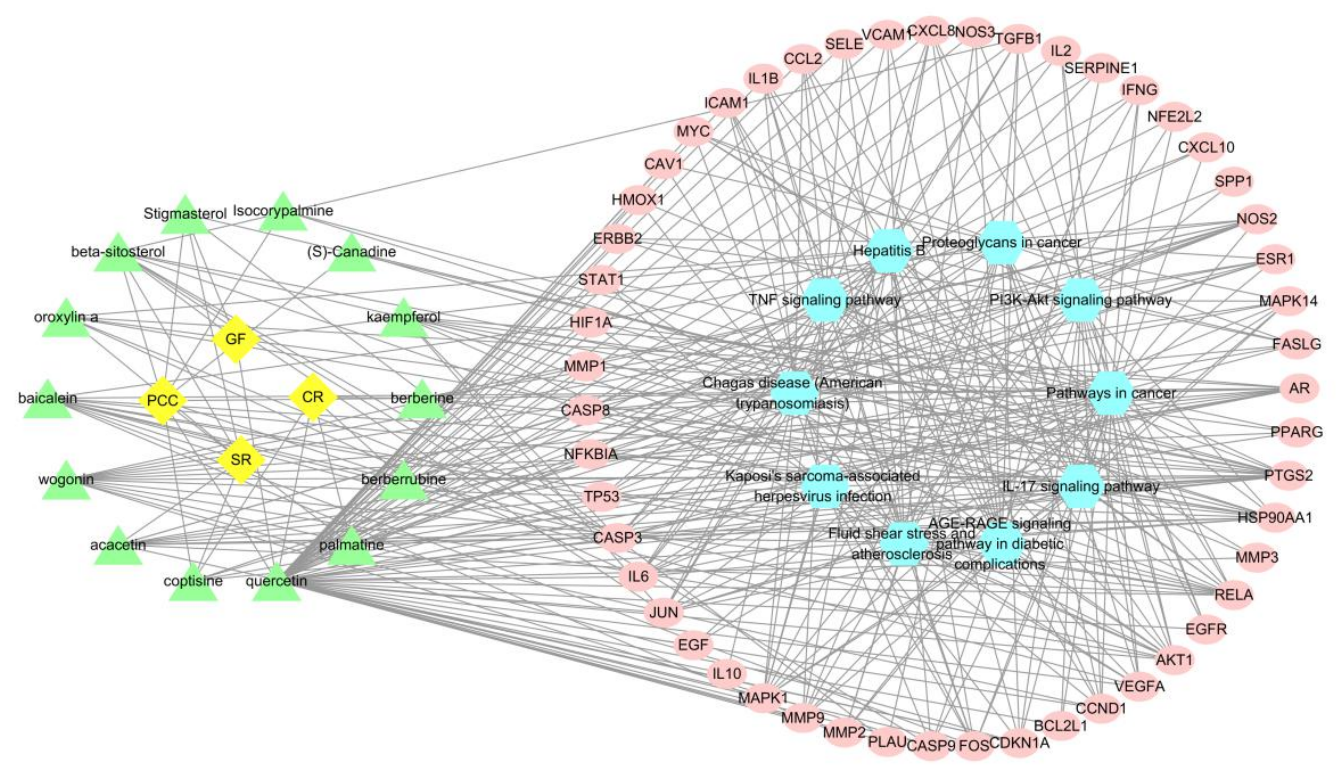

Figure7.HLJDD-Key Active Ingredients-Key Targets-Pathway Network of HLJDD for Anti-atherosclerosis

\section{Discussion}

In the field of TCM, HLJDD is a typical representative of heat-clearing and detoxifying prescription, which is often used to treat diseases caused by fire evil (TCM divides the pathogenic factors into six types, namely wind, cold, heat, wet, dry and fire). From the perspective of TCM, the incidence of atherosclerosis is related to the fire evil ${ }^{[24]}$. Therefore, HLJDD may have an anti-atherosclerotic effect, which has been confirmed in previous studies. In this study, we finally identified 14 key active ingredients, 65 key targets and 20 signaling pathways of HLJDD for anti-atherosclerosis, and constructed the pharmacological network of HLJDD for anti-atherosclerosis.The 14 key active ingredients are quercetin, beta-sitosterol, stigmasterol, kaempferol, wogonin, palmatine, baicalein,berberine, coptisine, isocorypalmine, acacetin, (S) -Canadine, berberrubine, oroxylin a, and they have corresponding relationship with 52 of 65 key targets, and may play an anti-atherosclerotic role by affecting the main 10 related signal pathways,which include Pathways in cancer, Kaposi's sarcoma-associated herpesvirus infection, Fluid shear stress and atherosclerosis, PI3K-Akt signaling pathway, IL-17 signaling pathway, AGE-RAGE signaling pathway in diabetic complications, Hepatitis B, Chagas disease (American trypanosomiasis), TNF signaling pathway, Proteoglycans in cancer. 
Of the 14 components, some have been shown to have anti-atherosclerosis effects in previous studies.With the most targets, it can be found from the literature that the research on the anti-atherosclerosis effect of quercetin is also the most extensive.Quercetin can improves blood vessel structure by reducing serum lipids, oxidative stress factors, aortic contraction, weight gain and blood pressure, prevents the formation of arterial plaques through antioxidant activity ${ }^{[25]}$,and fight atherosclerosis by enhancing autophagy in ApoE-/-mice ${ }^{[26]}$.Furthermore, in a high fructose-induced atherosclerosis mouse model, quercetin can attenuate atherosclerosis by inhibiting inflammation and apoptosis through the PI3K / AKT signaling pathway regulated by reactive oxygen species ${ }^{[27]}$. Both beta-sitosterol and stigmasterol belong to Phytosterols, and moderate intake of Phytosterols is considered to reduce plasma cholesterol and prevent atherosclerosis ${ }^{[28]}$.In addition, Lifsey $\mathrm{HC}$ et al reported that stigmasterol can promote the excretion of cholesterol in the small intestine ${ }^{[29]}$, while Chien $\mathrm{MH}$ et al reported that beta-sitosterol can inhibit the proliferation of vascular smooth muscle cells and has a potential role in anti-atherosclerosis ${ }^{[30]}$.In the rabbit model of atherosclerosis induced by high cholesterol, kaempferol can regulate the expression of inflammatory factors, showing an anti-atherosclerosis effect ${ }^{[31]} \mathrm{Ku}$ SK et al reported that wogonin and baicalein blocked hyperglycaemic vascular inflammation by inhibiting NF- $\mathrm{kB}$ in human primary endothelial cell models, suggesting that they had a therapeutic effect on atherosclerosis ${ }^{[32]}$.Shi $\mathrm{Y}$ et al reported that berberine can delay the progression of atherosclerosis in ApoE-/-mice induced by high-fat diet and reduce the expression of inflammatory factors, which may be related to the regulated intestinal flora ${ }^{[33]}$.Other studies have shown that berberine down-regulates visfatin expression to inhibit endothelial dysfunction and improve diet-induced atherosclerosis in ApoE-/- mice ${ }^{[34]}$.Feng $\mathrm{M}$ et al reported that in the atherosclerosis model of ApoE-/- mice, the intervention of coptisine can reduce the level of blood lipid and down-regulate the expression of relevant inflammatory cytokines, thus reducing the area of atherosclerotic plaque ${ }^{[35]}$.Although no previous studies have directly shown that palmatine, berberrubine, acetacetin, oroxylin a paly a role of anti-atherosclerosis, studies have shown that they all have different degrees of effects of anti-inflammatory, lipid-lowering, and anti-oxidation,et al.Berberrubine is the main metabolite of berberine in vivo. Studies have shown that it can reduce blood lipid, inhibit the activity of intracellular xanthine oxidase, and has a potential role in treating endothelial dysfunction ${ }^{[36-37]}$.Palmatine and oroxylin a both have anti-inflammatory effects, and the former can reduce blood lipid levels, while the latter can also inhibit intracellular lipid accumulation ${ }^{[38-39]}$.

As a flavonoid,Liou $\mathrm{CJ}$ et al reported that acacetin can reduce the expression of transcription factors related to adipogenesis, inhibit lipid accumulation in adipocytes, and inhibit the inflammatory response of macrophages stimulated by $3 \mathrm{~T} 3-\mathrm{L} 1$ cell differentiation medium ${ }^{[40]}$, while $\mathrm{Wu} \mathrm{WY}$ et al show that it can participate in anti-oxidation, anti-inflammatory and anti-apoptosis by activating AMPK-mediated Nrf2 pathway, which will play its protective effect on myocardial cell hypoxia / reoxygenation injury ${ }^{[41]}$.Based on the above research status, we speculate that the above chemical components are most likely related medicinal substances of HLJDD against atherosclerosis.

In terms of targets, in the final constructed pharmacological network, the top 3 nodes with the highest node degrees are PTGS2, HSP90AA1, and RELA.PTGS2 is connected to 14 component nodes and 4 pathway nodes, HSP90AA1 is connected to 11 component nodes and 4 pathway nodes, and RELA is connected to 5 component nodes and 9 pathway nodes. The PTGS2 gene 
encodes cyclooxygenase-2 (COX-2), and previous studies have shown that COX-2 is widely expressed in human atherosclerosis by monocytes, macrophages, medial smooth muscle cells, and endothelial cells ${ }^{[42-43]}$, which suggests that COX-2 may be involved in the formation and development of atherosclerosis.Atherosclerosis is a chronic inflammatory response process. In inflammatory response, $\mathrm{COX}-2$ expression can be rapidly induced by a variety of factors, such as IL-1 and TGF- $\beta$, while cox-2-induced prostaglandin production can promote inflammatory response of atherosclerosis. The activation of macrophages is one of the key mechanisms for the formation and development of atherosclerosis, and this process is related to the high expression of COX-2. The expression of COX-2 can promote the release and activation of matrix metalloproteinases, which play an important role in the migration of macrophages.Low density lipoprotein stimulates monocyte chemotaxis through the cox-dependent pathway.In general, Cox-2 expression induces prostaglandin production through activation of chemotaxis, induction of vascular permeability, cascade propagation of inflammatory cytokines, and stimulation of smooth muscle cell migration and proliferation,et al, thus promoting the formation and development of atherosclerosis ${ }^{[44]}$.HSP90AA1 is a subtype of the heat shock protein HSP90, and the overexpression of HSP90 in atherosclerotic plaques plays an important role in the maintenance of inflammation, which is related to the activation of nuclear factor- $\kappa \mathrm{B}(\mathrm{NF}-\kappa \mathrm{B})$ pathway. HSP90 folds and activates I $\mathrm{KB}$ kinase, which phosphorylates and induces the degradation of the proteasome inhibitor protein I $\mathrm{I} B \alpha$, so that the nuclear factor- $\kappa \mathrm{B}$ p 65 subunit enters the nucleus, promoting the inflammatory response and promoting the transcription of oxidative genes ${ }^{[45]}$. The RELA gene encodes Transcription factor p65, a subunit of the transcription factor family NF- $\mathrm{kB}$, which is a key regulator of eukaryotic inflammatory responses.Previous studies have shown that $\mathrm{NF}-\mathrm{KB}$ is closely related to the formation and development of atherosclerosis, which induces the expression of adhesion proteins, monocyte chemotactic protein, IL-1ßand other proinflammatory molecules in vascular endothelial cells to promote vascular inflammation ${ }^{[46]}$. The post-translational modification of RelA / p65 can finely regulate the transcriptional activation of NF- $\kappa$ B and play an important role in the occurrence and development of inflammatory response and diseases related to inflammatory response ${ }^{[47]}$. Furthermore, many previous studies have shown that other targets are closely related to the pathogenesis of atherosclerosis. For example, Interleukin-6, Interleukin-1ßand other pro-inflammatory factors are involved in the formation and development of atherosclerosis ${ }^{[48-49]}$, and the effects of matrix metalloproteinases such as MMP-1, MMP-3, and MMP-9 are closely related to the instability of atherosclerotic plaques ${ }^{[50]}$.All these previous studies provided certain theoretical support for our study. Therefore, we speculated that HLJDD might play an anti-atherosclerosis role by acting on 52 targets including PTGS2, HSP90AA1, RELA, IL-6, IL-1, MMP-1, MMP-3, and MMP-9.

In terms of pathways, KEGG pathway enrichment analysis results show that 65 key targets are also significantly enriched in diseases-related pathways such as herpes virus infection, hepatitis B, cancer, diabetes, and Chagas disease, suggesting that there may be correlations and effects between different diseases, Which has also been verified in previous research.Studies have shown that the hepatitis B core antibody (anti-HBc) positive / hepatitis B surface antigen (HBsAg) negative pattern is associated with intracranial atherosclerotic stenosis in hepatitis B virus infection ${ }^{[51]}$.In virologically suppressed HIV patients, human herpesvirus 8 (HHV-8) infection increases inflammation and activates immunity, increasing the risk of atherosclerosis ${ }^{[52]}$. With common risk factors, cancer and atherosclerosis have the same basis as metabolic or vascular 
abnormalities, and the theory of inflammation is the common pathological basis of both them ${ }^{[53]}$.Chronic inflammation caused by Chagas disease may trigger endothelial damage and platelet activation, accelerating atherosclerosis ${ }^{[54]} . \mathrm{In}$ the progression of atherosclerosis, the PI3K-Akt signaling pathway plays an important role in the survival, proliferation and migration of macrophages ${ }^{[5]}$, which suggesting that HLJDD may prevent atherosclerosis by regulating PI3K-Akt signaling pathway and inhibiting the related effects of macrophages.Signaling pathways such as IL-17 signaling pathway and TNF signaling pathway are closely related to inflammatory response. Activation of IL-17 signaling pathway and TNF signaling pathway can activate downstream NF- $\kappa$ B signaling pathways, leading to the production of various mediators associated with atherosclerosis, including inflammatory factors ,chemokines and others ${ }^{[6-57]}$. It is suggested that HLJDD may inhibit the production of inflammatory mediators by affecting the activation of il-17 signaling pathway, TNF signaling pathway and its downstream pathways, thus playing an anti-atherosclerosis role.Atherosclerosis is a common complication of diabetes. Activation of AGE-RAGE signaling pathway in diabetic complications participates in the formation and development of atherosclerosis. Advanced glycation endproducts (AGEs) binds to its receptor (RAGE) to activate a variety of intracellular signaling pathways such as NADPH oxidase, which eventually activates NF- $\mathrm{kB}$ and leads to the expression of pro-inflammatory factors such as IL-6 and TNF- $\alpha$, leading to atherosclerosis ${ }^{[58]}$, suggesting that HLJDD may prevent atherosclerosis by affecting the activation of AGE-RAGE signaling pathway in diabetic or its' downstream pathways and inhibiting the production of inflammatory factors.Hemodynamics is an important factor affecting atherosclerosis. Stable blood flow can prevent atherosclerosis by inhibiting oxidative stress and inflammation in the arterial wall,while disordered blood flow can promote oxidative stress and inflammatory response in the arterial wall to induce atherosclerosis, of which the mechanism is related to activation of endothelial cell activator protein 1 (AP-1) and nuclear factor kappaB (NF-kB) ${ }^{[59]}$, suggesting that HLJDD may inhibit the oxidation and inflammation of the arterial wall by improving hemodynamics and regulating the fluid shear stress and atherosclerosis signal pathway.

In summary,through the application of network pharmacology, we finally predicted 14 key active ingredients, 65 key targets and 10 pathways that may be related to the anti-atherosclerosis activity of HLJDD.HLJDD may affect 52 key targets such as PTGS2, HSP90AA1, and RELA through 14 key active ingredients such as quercetin, wogonin, and baicalein, and regulate Fluid shear stress and atherosclerosis, PI3K-Akt signaling pathway, IL-17 signaling pathway, AGERAGE signaling pathway in diabetic complications, TNF signaling pathway and other related signaling pathways, and then by inhibiting inflammatory response and oxidative stress, improving hemodynamics and other effects to prevent atherosclerosis.However, our results are only a prediction based on the results of previous studies, which need to be further verified by experimental research.

\section{Data Availability}

The data used to support the findings are included in the article and the supplementary files.

\section{Supplementary Materials}

Supplementary Table 1:102 Active Ingredients of HLJDD;Supplementary Table 2:222 Targets Corresponding to The Active Ingredients of HLJDD;Supplementary Table 3:3812 Related Targets 
of Atherosclerosis;Supplementary Figure1:PPI Analysis Network of 175 Targets.

\section{Conflict of Interest}

We declare that we have no competing interests.All authors have read and endorsed the manuscript.

\section{Funding}

This work is supported by The High-Level Key Disciplines Project of Guangzhou University of Chinese Medicine (No. A1-AFD018171Z11089 to DW).

\section{Author Contribution to Study}

Conception and design: Xia Yan,Dawei Wang

Experiment and data analysis: Kerui Wu,Lu Jiang,Lanlin Huang,Yaxing He

Paper writing: Kerui Wu

\section{Refrences:}

[1] Joseph P,Leong D,Mckee M,et al. Reducing the global burden of cardiovascular disease,Part 1: the epidemiology and risk factors[J]. Circ Res,2017,121(6):677-694.

[2] GBD 2015 Mortality and Causes of Death Collaborators. Global, regional, and national life expectancy, all-cause mortality, and cause-specific mortality for 249 causes of death, 1980-2015: a systematic analysis for the global burden of disease study 2015[J]. Lancet,2016,388:14591544.

[3] Mineo C.Lipoprotein Receptor Signaling in Atherosclerosis[J].Cardiovasc Res, 2019. pii: cvz338.

[4] Mitra R, Oneil GL, Harding IC, et al. Glycocalyx in atherosclerosis-relevant endothelium function and as a therapeutic target[J]. Curr Atheroscler Rep, 2017, 19(12): 63.

[5] Zhang RJ, Yin YF, Xie XJ,et al.Acid-sensing ion channels: Linking extracellular acidification with atherosclerosis[J].Clin Chim Acta,2019,502:183-190.

[6] Ziegler T, Abdel Rahman F, Jurisch V,et,al.Atherosclerosis and the Capillary Network; Pathophysiology and Potential Therapeutic Strategies[J].Cells,2019,9(1).pii: E50.

[7] Ross R.Atherosclerosis--an inflammatory disease[J].N Engl J Med,1999,340(2):115-126.

[8] Viola J, Soehnlein O. Atherosclerosis-A matter of unresolved inflammation[J]. Semin Immunol, 2015, 27(3): 184-193.

[9] Back M, Yurdagul A Jr, Tabas I, et al. Inflammation and its resolution in atherosclerosis: mediators and therapeutic opportunities[J]. Nat Rev Cardiol,2019,16(7):389-406.

[10] Chen Z, Gao X, Jiao Y,et al.Tanshinone IIA Exerts Anti-Inflammatory and Immune-Regulating Effects on Vulnerable Atherosclerotic Plaque Partially via the TLR4/MyD88/NF-אB Signal Pathway[J].Front Pharmacol, 2019,10:850.

[11] Li Q,Zhao W,Zeng X,et al.Ursolic Acid Attenuates Atherosclerosis in ApoE-/- Mice: Role of LOX-1 Mediated by ROS/NF-אB Pathway[J].Molecules,2018,23(5). pii: E1101.

[12] Gasmi A, Mujawdiya PK, Shanaida M,et,al.Calanus oil in the treatment of obesity-related low-grade inflammation, insulin resistance, and atherosclerosis[J].Appl Microbiol Biotechnol, 2019. doi: 10.1007/s00253-019-10293-4.

[13] Zhou FH,Cheng SB,Zhang Y,et al.nti-atherosclerotic effect of a Chinese medicine,Huanglian 
Jiedu Decoction,mediated by regulatory T cells[J].Acta Laboratorium Animalis Scientia Sinica,2016,24(3):233-238.

[14] Yu LB,Chen Y, Xu GL,et al.Study on Mechanism of Huang-Lian Jie-Du Decoction on Atherosclerosis Rats Based on Anti-inflammatory And Antioxidant[J].World Science and Technology/Modernization of Traditional Chinese Medicine and Materia Medica,2017,19(11): 1841-1845.

[15] Wang XW,Sang WF,Pei Q,et,al.Effects of Huanglian Jiedu Decoction on the rabbit atherosclerosis caused by toxic heatand endothelial progenitor cells[J].LI Shizhen Medicine And Materia Medica Research,2014,25(9):2121-2124.

[16] Xu Y,Qin M,Zhao Yk,et,al.The Effect of Cholesterol Lowing and Anti-atheroscl-erosis of Hanglian Jiedu Tang and Its Components[J].Chinese Journal of Experimental Traditional Medical Formulae,2008,14(2):74-77.

[17] Hopkins AL.Network pharmacology: the next paradigm in drug discovery[J].Nat Chem Biol,2008,4(11):682-690.

[18] Zhang GB, Li QY, Chen QL,et,al.Network pharmacology: a new approach for chinese herbal medicine research[J].Evid Based Complement Alternat Med, 2013,2013(8),Article ID621423.

[19] Zhao J,Fang HY, Zhang WD.Bioinformatics Approaches in Research on Network Pharmacology of Traditional Chinese Medicine[J].Progress in Pharmaceutical Sciences, 2014,38(2):97-103.

[20] Ru J, Li P, Wang J,et al.TCMSP: a database of systems pharmacology for drug discovery from herbal medicines[J].J Cheminform,2014,6:13. doi: 10.1186/1758-2946-6-13.

[21] Szklarczyk D, Gable AL, Lyon D,et al.STRING v11: protein-protein association networks withincreased coverage, supporting functional discovery in genome-wide experimental datasets [J].Nucleic Acids Res,2019,47(D1):D607-D613.

[22] Stelzer G, Rosen N, Plaschkes I,et al.The GeneCards Suite: From Gene Data Mining to Disease Genome Sequence Analyses[J].Curr Protoc Bioinformatics,2016,54:1.30.1-1.30.33.

[23] Amberger JS, Hamosh A.Searching Online Mendelian Inheritance in Man (OMIM): A Knowledgebase of Human Genes and Genetic Phenotypes[J].Curr Protoc Bioinformatics,2017,58: 1.2.1-1.2.12.

[24]Ding YQ,Qu J,Yang J.According to fire-heat theory to discuss pathogenesis and treatment of cervical atheroscleorsis[J].Shandong Journal of Traditional Chinese

Medicine,2007,26(3):147-149.

[25]Cao H, Jia Q, Shen D,et,al.Quercetin has a protective effect on atherosclerosis via enhancement of autophagy in ApoE-/-mice[J].Exp Ther Med, 2019,18(4):2451-2458.

[26]Parvin A, Yaghmaei P, Noureddini M,et,al.Comparative effects of quercetin and hydroalcoholic extract of Otostegia persica boiss with atorvastatin on atherosclerosis complication in male wistar rats[J].Food Sci Nutr, 2019,7(9):2875-2887.

[27]Lu XL, Zhao CH, Yao XL,et al.Quercetin attenuates high fructose feeding-induced atherosclerosis by suppressing inflammation and apoptosis via ROS-regulated PI3K/AKT signaling pathway[J].Biomed Pharmacother, 2017,85:658-671.

[28]Cabral CE, Klein MRST.Phytosterols in the Treatment of Hypercholesterolemia and Prevention of Cardiovascular Diseases[J].Arq Bras Cardiol, 2017,109(5):475-482.

[29]Lifsey HC, Kaur R, Thompson BH,et al.Stigmasterol stimulates transintestinal cholesterol excretion independent of liver X receptor activation in the small intestine[J].J Nutr Biochem,2019, 
76:108263.

[30] Chien MH, Lee TS, Liang YC,et al. $\beta$-Sitosterol inhibits cell cycle progression of rat aortic smooth muscle cellsthrough increases of p21cip1 protein[J].J Agric Food

Chem.2010,58(18):10064-9.

[31]Kong L, Luo C, Li X,et al.The anti-inflammatory effect of kaempferol on early atherosclerosis in high cholesterol fed rabbits[J].Lipids Health Dis,2013,12:115.

[32]Ku SK, Bae JS.Baicalin, baicalein and wogonin inhibits high glucose-induced vascular inflammation in vitro and in vivo[J].BMB Rep,2015,48(9):519-524.

[33]Shi Y, Hu J, Geng J,et al.Berberine treatment reduces atherosclerosis by mediating gut microbiota in apoE-/- mice[J].Biomed Pharmacother, 2018,107:1556-1563.

[34]Wan Q, Liu Z, Yang Y,et al. Suppressive effects of berberine on atherosclerosis via downregulating visfatin expression and attenuating visfatin-induced endothelial dysfunction[J]. Int J Mol Med,2018,41(4):1939-1948.

[35]Feng M, Kong SZ, Wang ZX,et al.The protective effect of coptisine on experimental atherosclerosis ApoE-/- mice is mediated by MAPK/NF- $\kappa \mathrm{B}-$ dependent pathway[J].Biomed Pharmacother,2017,93:721-729.

[36]Cao S, Xu P, Yan J, et al.Berberrubine and its analog, hydroxypropyl-berberrubine, regulate LDLR and PCSK9 expression via the ERK signal pathway to exert cholesterol-lowering effects in human hepatoma HepG2 cells[J].J Cell Biochem,2018. doi: 10.1002/jcb.27102.

[37]Porru E, Franco P, Calabria D,et al.Combined analytical approaches to define biodistribution and biological activity of semi-synthetic berberrubine, the active metabolite of natural berberine[J].Anal Bioanal Chem,2018,410(15):3533-3545.

[38] Tarabasz D, Kukula-Koch W.Palmatine:A review of pharmacological properties and Pharmacokinetics[J].Phytother Res,2019. doi: 10.1002/ptr.6504.

[39]Lu L, Guo Q, Zhao L.Overview of Oroxylin A:

A Promising Flavonoid Compound[J].Phytother Res,2016,30(11):1765-1774.

[40]Liou CJ, Wu SJ, Chen LC,et al.Acacetin from Traditionally Used Saussurea involucrata Kar. et Kir. Suppressed Adipogenesis in 3T3-L1 Adipocytes and Attenuated Lipid Accumulation in Obese Mice[J].Front Pharmacol,2017,8:589.

[41]Wu WY, Li YD, Cui YK,et al.The Natural Flavone Acacetin Confers Cardiomyocyte Protection Against Hypoxia/Reoxygenation Injury via AMPK-Mediated Activation of Nrf2 Signaling Pathway[J].Front Pharmacol,2018,9:497.

[42]Schonbeck U, Sukhova GK, Graber P, et al. Augmented expression of cyclooxygenase-2 in human atherosclerotic lesions[J]. Am J Pathol,1999,155:1281-1291.

[43]Baker CS, Hall RJ, Evans TJ, et al. Cyclooxygenase-2 is widely expressed in atherosclerotic lesions affecting native and transplanted human coronary arteries and colocalizes with inducible nitric oxide synthase and nitrotyrosine particularly in macrophages[J]. Arterioscler Thromb Vasc Biol,1999,19:646-655.

[44]MacRae F , Serg io F.Cyclooxygenase-2 and atherosclerosis[ J].Curr Opin Lipidol,2002,13:497-504 .

[45]Lazaro I, Oguiza A, Recio C,et al.Interplay between HSP90 and Nrf2 pathways in diabetes -associated atherosclerosis[J].Clin Investig Arterioscler,2017,29(2):51-59.

[46]Van der Heiden K, Cuhlmann S, Luong LA, Zakkar M, Evans PC. Role of nuclear factor kappaB in cardiovascular health and disease[J]. Clin Sci(Lond),2010,118:593-605. 
[47]LI YB,CAI JW,LIU JH.Post-translational Modification of RelA / p65 in Regulating NF-кB Transcriptional Activities[J].Chinese Journal of Biochemistry and Molecular Biology,2012,28(11): 977-983.

[48]Hartman J, Frishman WH.Inflammation and atherosclerosis: a review of the role of interleukin- 6 in the development of atherosclerosis and the potential for targeted drug therapy[J].Cardiol Rev,2014,22(3):147-151.

[49]Libby P.Interleukin-1 Beta as

a Target for Atherosclerosis Therapy: Biological Basis of CANTOS and Beyond[J].J Am Coll Cardiol,2017,70(18):2278-2289.

[50]Silvello D, Narvaes LB, Albuquerque LC, et al.Serum levels and polymorphisms of matrix metalloproteinases(MMPs) in carotid artery atherosclerosis: higher MMP-9 levels are associated with plaquevulnerability[J].Biomarkers,2014,19(1):49-55.

[51]Shen L, Zhou H, Wei F,et al.The hepatitis B core antibody positive/hepatitis B surface antigen negative pattern is associated with the increased risk of intracranial atherosclerotic stenosis[J]. Medicine (Baltimore),2020,99(2):e18752.

[52]Masiá M, Robledano C, Ortiz de la Tabla V,et al.Coinfection with human herpesvirus 8 is associated with persistent inflammation and immune activation in virologically suppressed HIV-infected patients[J].PLoS One,2014,9(8):e105442.

[53]Raposeiras Roubín S, Cordero A.The Two-way Relationship Between Cancer and Atherosclerosis[J].Rev Esp Cardiol (Engl Ed),2019,72(6):487-494.

[54]Oliveira-Filho J.Stroke and brain atrophy in chronic Chagas disease patients: A new theory proposition[J].Dement Neuropsychol, 2009,3(1):22-26.

[55]Linton MF, Moslehi JJ, Babaev VR.Akt Signaling in Macrophage Polarization, Survival, and Atherosclerosis[J].Int J Mol Sci,2019,20(11). pii: E2703.

[56]Taleb S, Tedgui A, Mallat Z.IL-17 and Th17 cells in atherosclerosis: subtle and contextual roles[J].Arterioscler Thromb Vasc Biol,2015,35(2):258-264.

[57]Aggarwal BB, Shishodia S, Takada Y,et al.TNF blockade: an inflammatory issue[J].Ernst Schering Res Found Workshop,2006,56:161-186.

[58]Del Turco S, Basta G.An update on advanced glycation endproducts and atherosclerosis[J]. Biofactors,2012,38(4):266-274.

[59]Nigro P,Abe J,Berk BC.Flow shear stress and atherosclerosis: a matter of site specificity[J]. Antioxid Redox Signal,2011,15(5):1405-1414. 
Figures

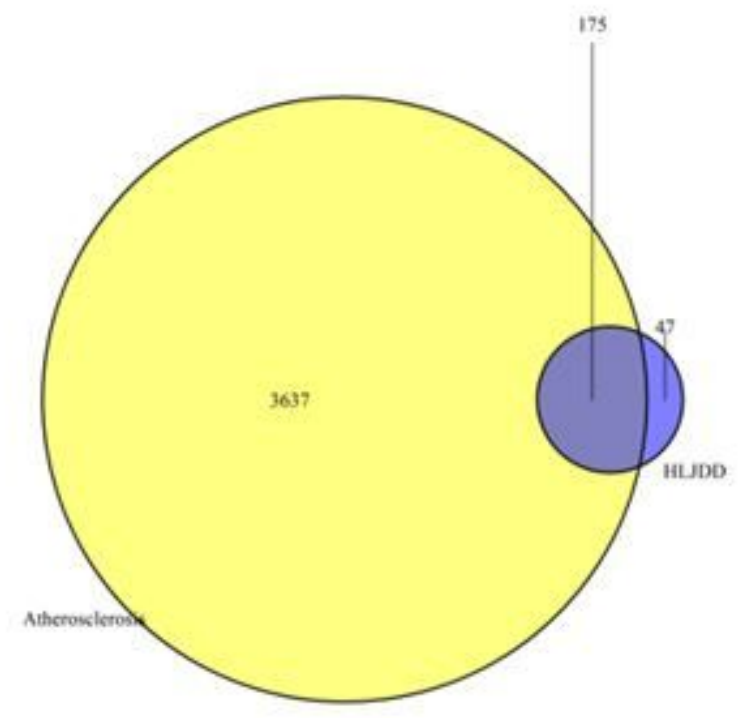

Figure 1

Vene figure of Screening Potential Targets of HLJDD for Anti-atherosclerosis 


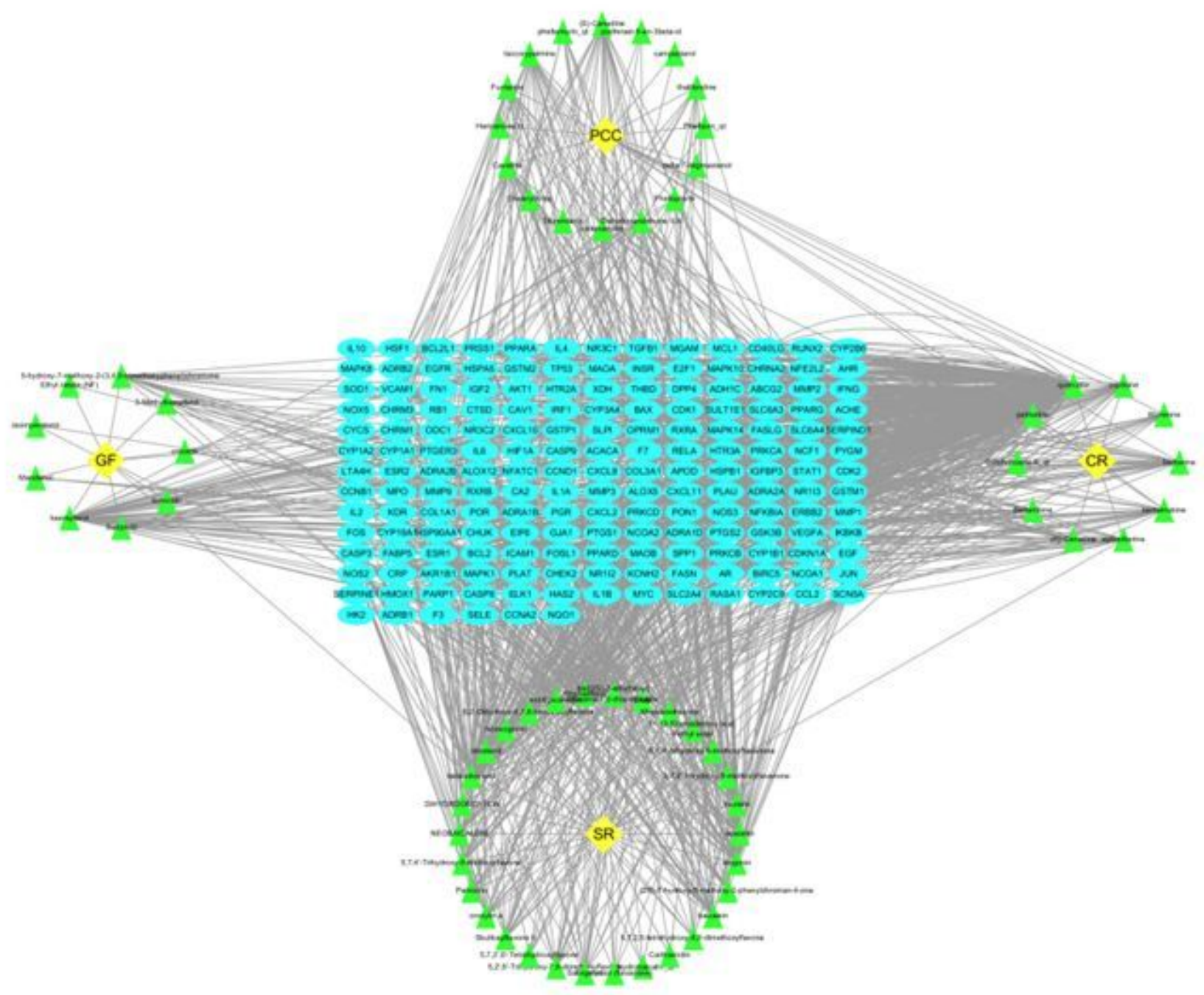

Figure 2

HLJDD-Active Ingredient-Target Regulation Network 


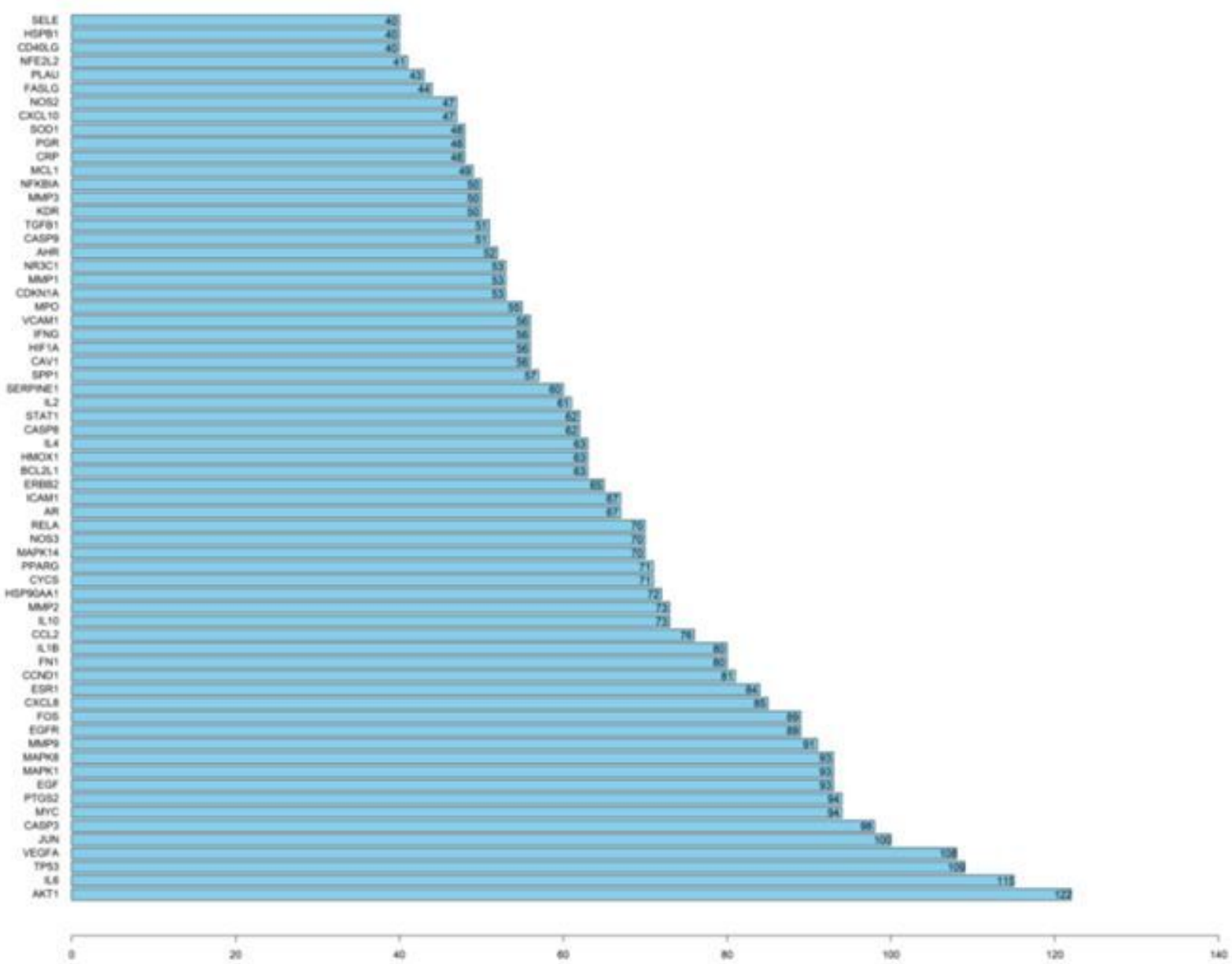

Figure 3

65 Key Targets and Their Node Connection Degree 


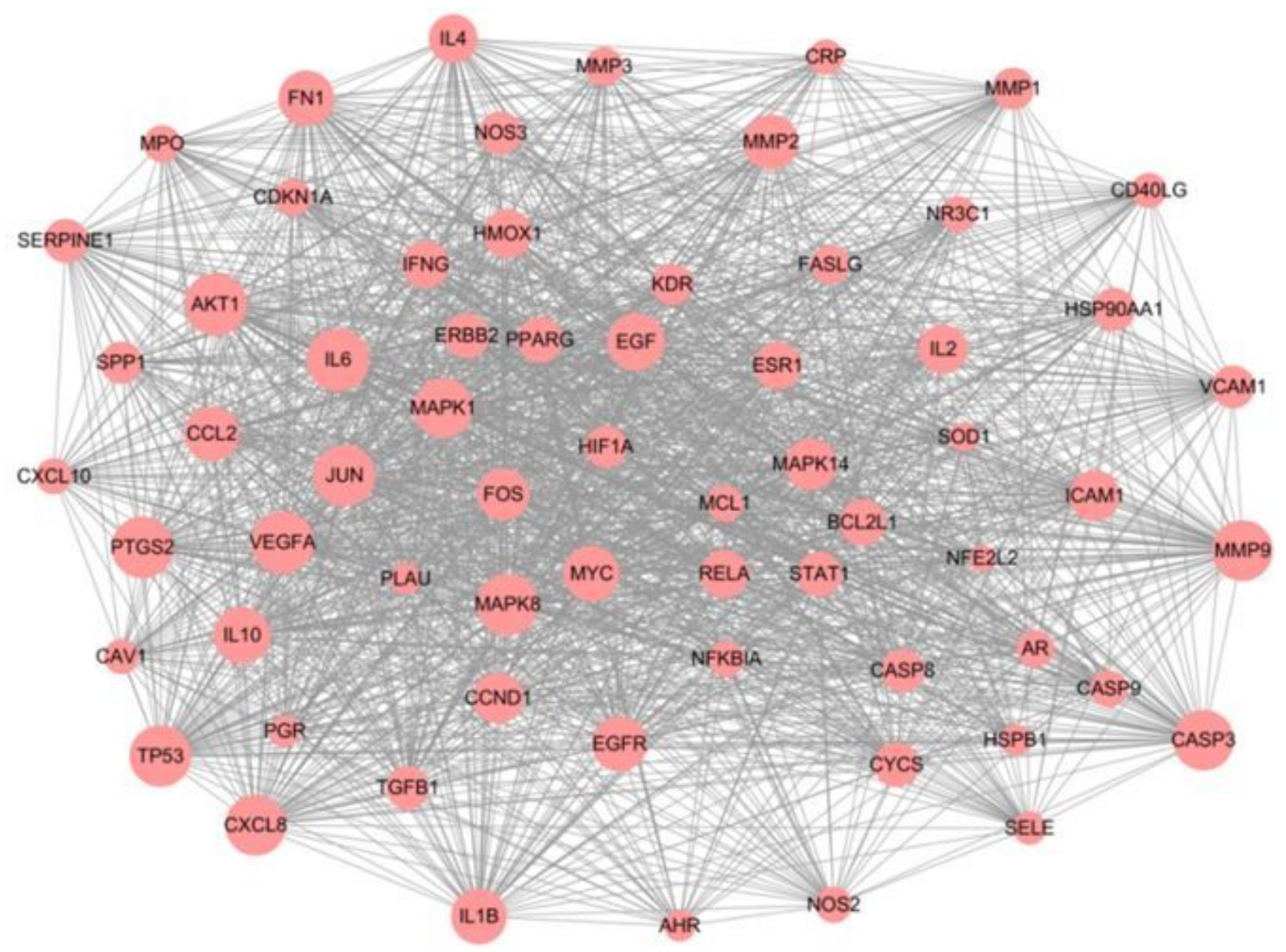

Figure 4

PPI Network of Key Targets of HLJDD for Anti-atherosclerosis 


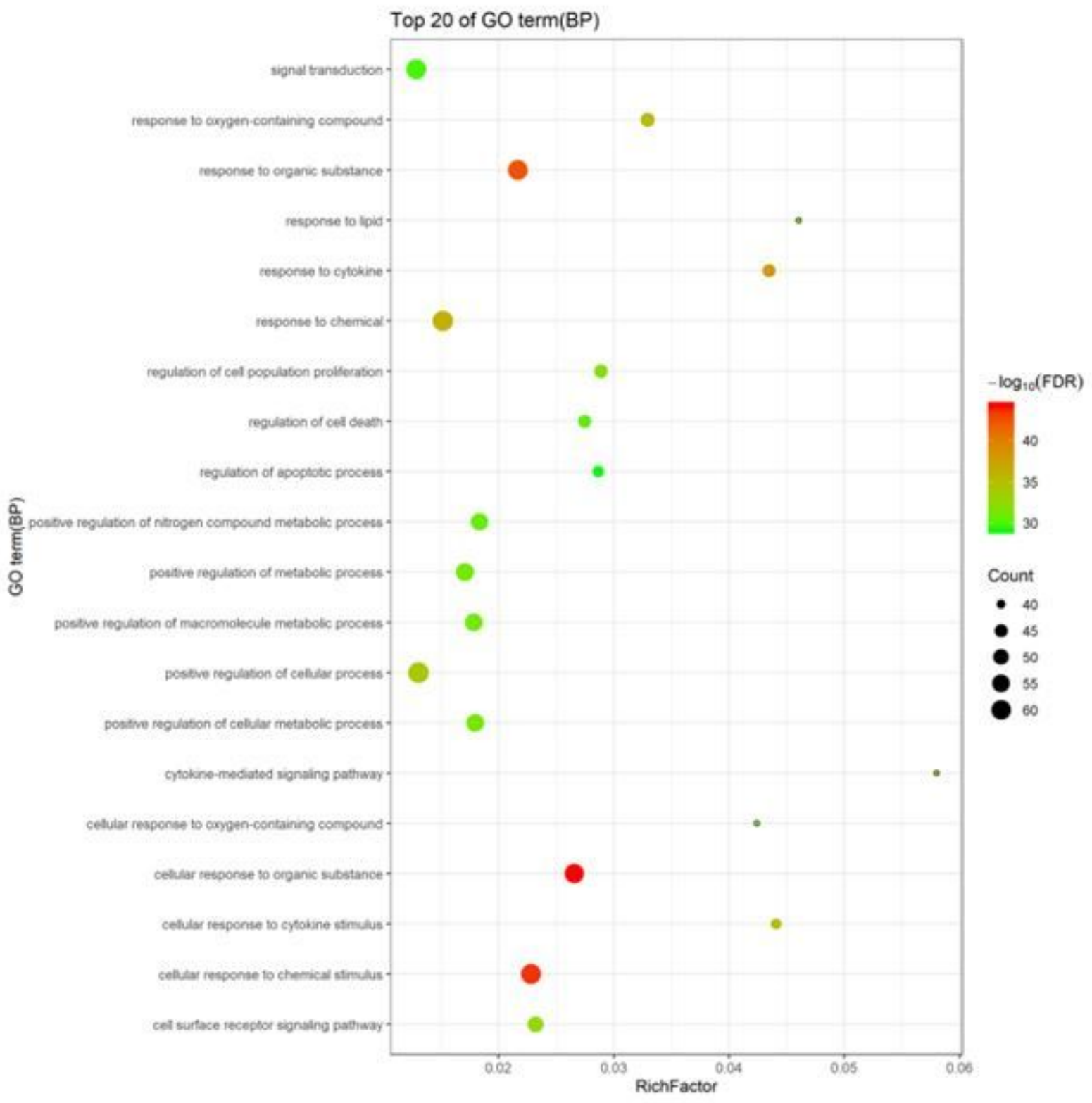

Figure 5

GO Analysis(Biological Process) of Key Targets of HLJDD for Anti-atherosclerosis 


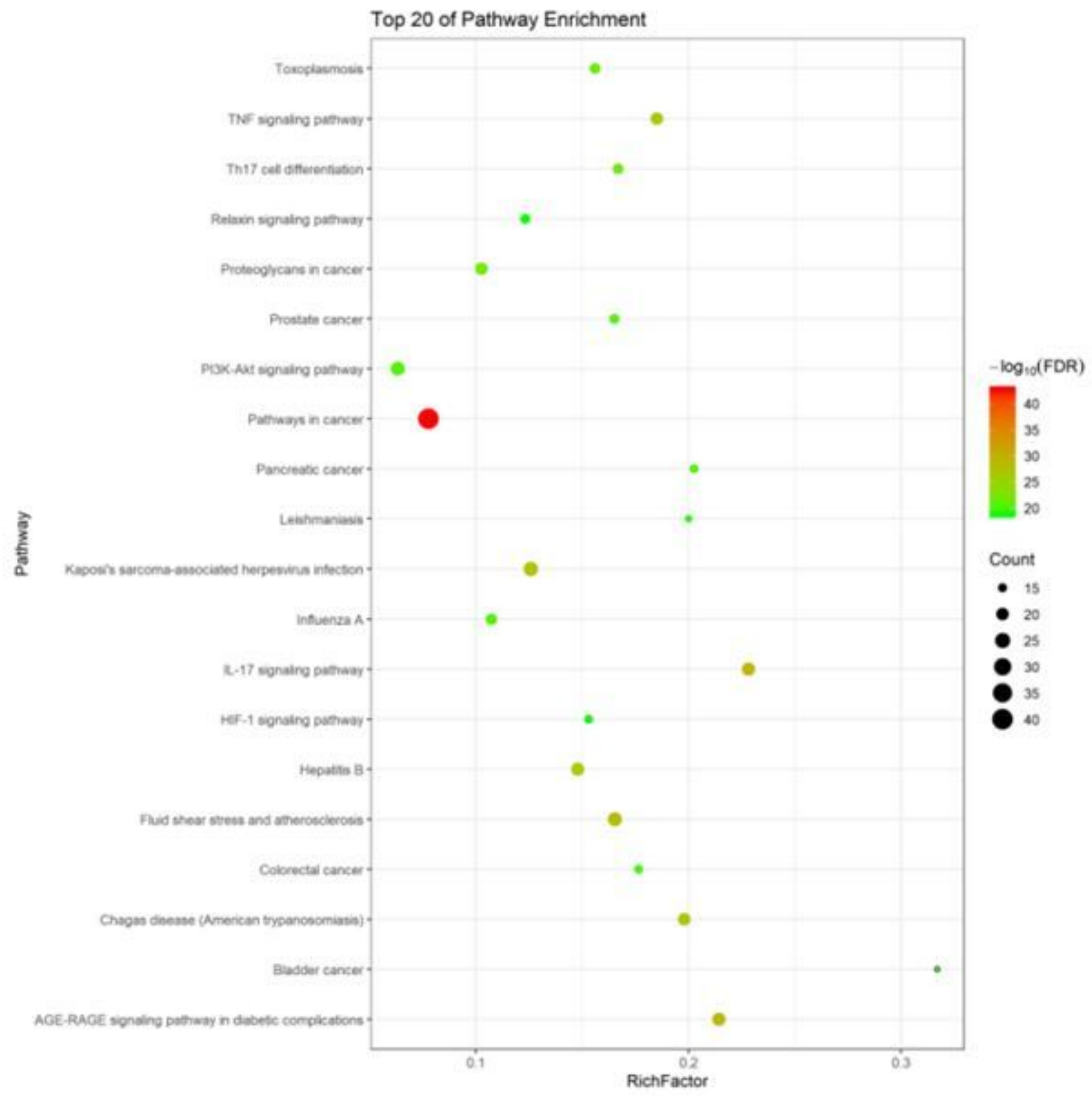

Figure 6

KEGG Pathway Enrichment Analysis of Key Targets of HLJDD for Anti-atherosclerosis 


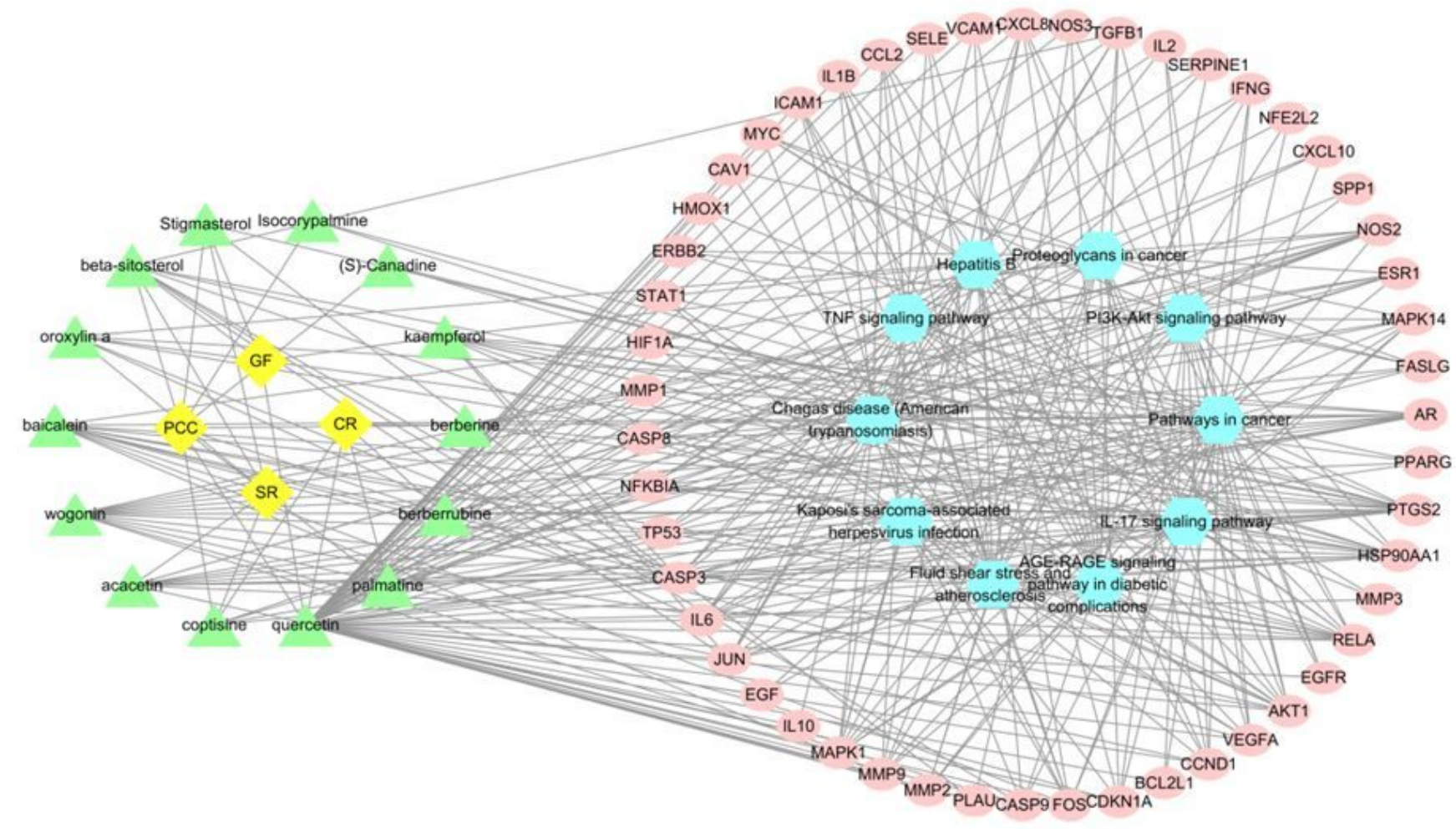

Figure 7

HLJDD-Key Active Ingredients-Key Targets-Pathway Network of HLJDD for Anti-atherosclerosis

\section{Supplementary Files}

This is a list of supplementary files associated with this preprint. Click to download.

- SupplementaryTable2.xls

- SupplementaryTable1.xls

- SupplementaryTable3.xls

- SupplementaryFigure1.docx 